\title{
Die „sympathische Arthritis“ - ein Symptom der Chronischen rekurrierenden multifokalen Osteomyelitis (CRMO)
}

F. Jakob

${ }^{1}$ Rheinland-Pfälzisches Rheuma-Zentrum Mainz/Bad Kreuznach

2 Klinik und Poliklinik für Radiologie der Johannes Gutenberg-Universität Mainz

${ }^{3}$ Orthopädische Klinik der Johannes Gutenberg-Universität Mainz

${ }^{4}$ Anatomisches Institut der Johannes Gutenberg-Universität Mainz

\section{Erratum}

In Heft 2 der Aktuellen Rheumatologie (F. Schilling et al. 2001; 26: 61 -74) kam es auf Seite 61 zu einem sinnverfälschenden Druckfehler. Unter dem Motto zur Bone and Joint Decade muss es heißen: „Wann und wie kommt Entzündung vom Knochen ins Gelenk?“ Wir bitten dieses Versehen zu entschuldigen. 